\title{
Mixed Effect Modeling in Analgesia Trials
}

Steven L. Shafer, MD*

Michel M. R. F. Struys, MD+ wo articles in this month's issue of Anesthesia \& Analgesia present novel analgesic concepts. The paper by Aasvang et al. ${ }^{1}$ explores the effect of instilling capsaicin into the wound during surgery. In the absence of some form of anesthesia, the capsaicin would be intensely painful. However, since the wound is also anesthetized using local, regional, or general anesthesia, the patient does not experience the immediate pain from C-fiber activation. Instead, $C$ fibers innervating the wound are rendered inactive from the calcium influx, providing long lasting postoperative analgesia. Also in this issue, the paper by Zeidan et al. ${ }^{2}$ demonstrates the analgesic utility of instilling a combination of tramadol and bupivacaine into the knee following arthroscopic knee surgery. The combination provides superior analgesia to either drug given alone.

What is unique about these papers is that the analgesic response was analyzed using a program unfamiliar to most analgesic investigators: NONMEM. ${ }^{3}$ NONMEM is a software program for nonlinear mixed effects modeling developed by Lewis Sheiner and Stuart Beal at the University of California. NONMEM was primarily developed to analyze pharmacokinetic data, and grew out of the recognition that pharmacokinetic data typically have two sources of variability: interpatient variability (how each patient's volumes and clearances were different from a "typical" patient) and residual intrasubject variability (assay noise, model misspecification, and other sources of noise). The bifurcation of variability into true biological variability, how we all differ from each other, and residual noise ushered in an era of individualized pharmacokinetics and therapeutic drug monitoring.

The authors of these two papers did not independently decide to analyze their data using NONMEM. Instead, their initial submissions reflected more traditional analyses that did not take full advantage of the repeated measures in their study design. For example, in the original submission on the effect of capsaicin, the analgesic effect was reported as a trend that did not reach statistical significance. Visual examination of the data during peer review clearly demonstrated that the groups were different at every point in time. The inability to distinguish this in the analysis was an artifact of the statistical approach.

Mixed effects analysis with NONMEM brought two unique aspects to the data analysis of these trials. Since pain is highly subjective, subjects differ enormously in their sensitivity to pain and their response to analgesics. A statistical approach must be able to isolate and characterize intersubject variability. Second, the analgesic response is not constant, but follows its own unique time course. Does the analgesia last for one day? Two days? Three days? Four days? Is the effect the same each day, or does it change over time?

These characteristics play to NONMEM's strengths. NONMEM can analyze intersubject variability (i.e., the unique sensitivity of subjects to pain and pain relief), and permits the analyst to precisely characterize the time course of the analgesic response. The model need not look like a pharmacokinetic model. Indeed, the model for both analgesics was a simple decrement in visual analog scale score that persisted over several days. 
The net result was that the data were allowed to forcefully speak to the underlying hypothesis. In the case of wound instillation of capsaicin, the result went from not being statistically significant, to being a large effect (about $50 \%$ reduction in visual analog scale score) that was significant at $P<0.0001$ ! The "traditional" analysis was essentially blind to an effect that was visually obvious and enormously statistically significant once interindividual variability was properly factored into the model.

The lesson to be learned is that mixed effect models should become the standard approach for analysis of analgesic trials. Mixed effect models can be implemented with many programs, including SAS, R, and S plus. However, NONMEM is optimized for such approaches, has been vetted by over two decades of testing, and has thousands of active users able to help investigators implement these approaches.

\section{REFERENCES}

1. Aasvang EK, Hansen JB, Malmstrøm J, Asmussen T, Gennevois $\mathrm{D}$, Struys MMRF, Kehlet $\mathrm{H}$. The effect of wound instillation of a novel purified capsaicin formulation on postherniotomy pain: a double-blind, randomized, placebo controlled study. Anesth Analg 2008;107:282-91

2. Zeidan A, Kassem R, Nahleh N, Maaliki H, El-khatib M, Struys MMRF, Baraka A. Intraarticular tramadol - bupivacaine combination prolongs the duration of postoperative analgesia after outpatient arthroscopic knee surgery. Anesth Analg 2008;107:292-9 3. Beal SL, Sheiner LB, NONMEM User Guides. NONMEM Project Group, UCSF, San Francisco, 1992 